\title{
Norois
}

Environnement, aménagement, société

222 | 2012

Xynthia

\section{Le droit dans la tempête}

The law in the storm

\section{Patrick Le Louarn}

\section{(2) OpenEdition}

\section{Journals}

Édition électronique

URL : https://journals.openedition.org/norois/3912

DOI : 10.4000 /norois.3912

ISBN : 978-2-7535-1843-8

ISSN : 1760-8546

\section{Éditeur}

Presses universitaires de Rennes

\section{Édition imprimée}

Date de publication : 28 février 2012

Pagination : 61-77

ISBN : 978-2-7535-1815-5

ISSN : 0029-182X

\section{Référence électronique}

Patrick Le Louarn, «Le droit dans la tempête », Norois [En ligne], 222 | 2012, mis en ligne le 30 mars 2014, consulté le 13 janvier 2022. URL : http://journals.openedition.org/norois/3912 ; DOI : https:// doi.org/10.4000/norois.3912 


Déseau des Universités

\title{
Le droit dans la tempête
}

\author{
The law in the storm
}

\author{
Patrick Le LouARN
}

\begin{abstract}
UMR 3128 CNRS, Laboratoire Droit et Changement Social, Université de Nantes, Faculté de droit et des sciences politiques, Chemin de La Censive-du-Tertre, BP 81307 - 44313 NANTEs Cedex 3, France (patrick.lelouarn@ univ-rennes $2 . f r$
\end{abstract}

Résumé : En détruisant maisons et digues, en tuant plusieurs personnes, la tempête Xynthia a révélé les lacunes du droit français en matière de prévention et de réparation des dommages dus aux submersions marines. Le découplage de la solidarité nationale et des assurances, la mauvaise coordination des documents d'urbanisme et des plans de prévention des risques incitent trop de personnes à s'installer dans les zones dangereuses. L'absence d'une véritable gestion intégrée des risques naturels sur le littoral aggrave les risques d'exposition d'un grand nombre d'habitants aux dangers naturels majeurs.

Le juge déterminera les responsabilités mais l'État ne pourra pas faire l'économie d'une amélioration de ses dispositifs législatifs et matériels pour éviter la reproduction de tels effets d'événements climatiques dont le retour est à prévoir. L'institution de plan de gestion des inondations dans les bassins versants par la loi dite Grenelle II est un premier pas en ce sens.

Abstract: By destroying houses and dikes, by killing several persons, the storm Xynthia revealed the gaps of the French law in prevention and in repair of the damage due to the marine floods. The decoupling of the national solidarity and the insurances, the bad coordination of the documents of town planning and the plans of risk prevention incite too many persons to settle down in the danger zones. The absence of real one management integrated by the natural risks on the coast aggravates the risks of exposure of inhabitants' large number in the major natural dangers.

The judge will determine the responsibilities but the State cannot save an improvement of its legislative and material devices to avoid the reproduction of such effects of climate-related events the return of which is to be planned. The institution of plan of management of the floods in ponds hillsides by the said law Grenelle II is the first step on this way.

Mots clé : assurances - documents d'urbanisme - domaine public - permis de construire - risques naturels - solidarité nationale.

Keywords : insurances - documents of town planning - public domain - building permit - natural risks - national solidarity 


\section{INTRODUCTION}

Though his bark cannot be lost Yet it shall be tempest-tost.

(Bien que son esquif ne puisse être perdu Il sera encore secoué par la tempête.)

\section{William Shakespeare (Macbeth)}

Dans les temps bibliques les catastrophes naturelles révélaient aux hommes le jugement divin sur leurs turpitudes. Xynthia aurait pu figurer dans la liste prestigieuse des dix plaies d'Égypte. Si notre connaissance scientifique de la nature en écarte désormais toute lecture magique ou religieuse, ce fait naturel garde toute sa force de révélateur. On en voudra pour preuve le débat de coupole académique sur le rapport entre Xynthia et un réchauffement climatique qui n'est autre que la résultante globale des turpitudes humaines à l'égard de la planète (Séchet, 2004).

Xynthia est un formidable révélateur de faits tangibles qui interrogent le droit dans la mesure où le droit cristallise les responsabilités et organise la maîtrise des risques dans un rapport tendu entre les moyens de l'autorité publique et « une société impatiente d'être protégée »(Auby, 2005). Il est donc naturel de penser que les enjeux juridiques posés par la tempête constituent un décalque de tous les éléments de l'exposition au risque majeur et de ses conséquences humaines et matérielles. Mais ce ne serait pas suffisant car l'enjeu porte sur le droit lui-même ou plutôt sur sa lecture et sa pratique comme le laissait entendre Auby dans un exposé prémonitoire en 2005. En effet, les dimensions parfois incommensurables du problème et sa répétition probable obligent à faire un tri entre les intérêts, à relire le partage des responsabilités avec d'autres lunettes que celles de nos règles les plus éprouvées, à redéfinir l'autorité publique et son territoire d'application, à repenser la solidarité nationale et la solidarité civile des assurés, à revoir les outils de gestion du risque et leurs procédures d'élaboration... Les pouvoirs publics et le droit ne sortiront pas davantage indemnes de l'événement que les malheureuses victimes de la tempête.

L'événement révèle en effet tous les ingrédients de l'exposition à la catastrophe que constitue la submersion marine et dont le rapport de la mission parlementaire dresse l'inventaire (Anziani, 2010).

La société française n'a pas intégré la culture des risques naturels tout au contraire de la société néerlandaise, par exemple. Il est vrai que les enjeux économiques de la façade atlantique ne sont pas assez importants pour justifier proportionnellement les dépenses d'une protection continue sur un linéaire considérable. Mais l'attractivité du littoral, avec une croissance démographique deux fois plus élevée que sur le reste du territoire, augmente considérablement l'exposition des biens et des personnes aux dangers naturels dont on devrait s'éloigner. De plus, le désordre territorial interdit de définir un territoire du risque qui serait le territoire de la prévention et d'une autorité publique qui en serait chargée. Enfin, les réponses publiques et privées au risque encouru sont inadéquates car, non seulement elles ne protègent pas des conséquences les plus graves mais elles aggravent l'exposition au danger : spéculation foncière, légèreté de la gestion des digues et sous estimation du danger par le zonage urbain, inadéquation des réponses publiques par un zonage de prévention ou de réparation dont les objectifs sont ambigus, recherche de compromis des intérêts qui néglige la puissance du retour d'événement, conception unilatérale du droit de propriété qui néglige le caractère réellement composite de la propriété foncière exprimant la solidarité entre le propriétaire et les assureurs, les fonds d'État, l'autorité de police, les promoteurs immobiliers, les élus locaux, etc.

La Mission parlementaire a constaté que les acteurs locaux de l'aménagement ont parfois engagé bien légèrement leur responsabilité en faisant souscrire à leurs partenaires des enjeux beaucoup plus importants que ceux-ci ne pouvaient le penser. Ainsi, le bénéficiaire d'un permis de construire peut croire acquérir une rente productive de plus-value alors qu'il assume le risque absolu de destruction en première ligne y compris pour sa vie. Mais son assureur et, derrière celui-ci, la solidarité des assurés plus la solidarité nationale, sont engagés par la quantité de biens et de vies détruits avec un montant global de réparations considérables et pour certaines impossibles. L'intérêt commun de ce groupe très hétérogène directement ou indirectement atteint que l'on peut appeler : « les victimes », est donc de faire contribuer à la réparation ceux qu'on ne peut pas appeler les auteurs - car l'auteur c'est 
Xynthia - mais qui ont, soit autorisé l'exposition au risque, soit organisé celle-ci en mettant les biens sur le marché. L'événement nous contraindra ainsi à une répartition des responsabilités qui ne sera pas exactement calquée sur le partage initial des enjeux et devra mesurer les participations de chacun au malheur général.

Les arbitrages de la réparation mettront en œuvre plusieurs outils juridiques : les systèmes d'indemnisation, dont les faiblesses sont connues, la planification et les procédures de prévention, conduites par les préfets et les maires, le juge qui n'interviendra que sur des demandes individuelles, et le Parlement dont le rôle est de présenter une description objective des faits et des responsabilités dans une perspective de leçon pour l'avenir et de réforme législative. Tous nos systèmes d'arbitrage entre les responsabilités sont, en effet, convoqués par une telle tempête : responsabilité civile, socialisation du risque, responsabilité assurantielle, responsabilité pénale, responsabilité administrative, etc. Cette communication n'a pas pour objet d'analyser toutes les responsabilités une fois dite leur importance. Car on verra certainement le juge alimenter nos réflexions à venir mais aussi parce que la question d'aujourd'hui est de dresser le tableau des enjeux mis au jour par un tel événement. Enjeux que l'on évalue dans un contexte où la seule incertitude porte sur la reproductibilité de l'événement.

Mais de quelle nature sont ces enjeux qui impliquent toute la chaîne des acteurs? On peut les regrouper en deux catégories : les enjeux liés à la réparation (i) qui répartit la charge entre les victimes, les responsables et la solidarité nationale et les enjeux de la prévention (ii) qui passe par des dépenses de protection mais aussi des contraintes et des servitudes qui feront supporter partiellement la charge par les propriétaires et habitants des zones menacées.

Une réparation totale et une prévention absolue sont-elles possibles? Il nous est permis d'en douter et cette part de l'irréparable, payée en vie humaines et en dégradation de patrimoine, ne peut qu'accroître la valeur des enjeux en cause. Il faut donc, pour chaque ensemble d'enjeux, faire un constat et imaginer les réformes nécessaires, non seulement la réforme des politiques publiques et de la gouvernance territoriale mais aussi la réforme des textes et des mécanismes juridiques qui assurent l'équilibre des responsabilités, la règle de droit devenant elle-même un des enjeux révélés par Xynthia ${ }^{1}$. En effet, la situation et l'imbrication des enjeux est si complexe qu'il faut aussi s'intéresser aux mécanismes qui les déclenchent, non pas le mécanisme naturel mais les mécanismes institutionnels et juridiques, concernant la gestion des territoires et la répartition des responsabilités (Favro, 2009), qui font qu'un maximum de risques est pris par un maximum de personnes qui n'en ont pas fait le choix aussi librement qu'on peut le penser. Dans ce jeu, l'autorité publique, avec ses outils de droit public, joue le rôle principal.

Sans avoir à le développer on doit évoquer d'emblée l'enjeu moral, philosophique et politique qui domine la question de l'exposition aux risques de submersion marine. Peut-on, en effet, dans le contexte d'une Europe particulièrement exposée aux aléas naturels ${ }^{2}$, espérer mettre d'accord victimes, payeurs et propriétaires sur une modification des règles de propriétés, d'occupation de l'espace et de l'assurance, de délimitation des pouvoirs de la puissance publique, qui diminueraient l'exposition aux risques et organiseraient une répartition plus juste des charges et des responsabilités?

\section{LES ENJEUX LIÉS À LA RÉPARATION}

Bien que les coûts soient considérables, la réparation matérielle ne semble pas insurmontable alors que les pertes humaines sont insupportables et démontrent que l'exposition aux risques est trop forte sur notre littoral atlantique. Ainsi c'est moins le coût de la réparation qu'il faut considérer que les principes de protection de la vie humaine et de justice dans la répartition de la charge qui va atteindre l'ensemble de la Nation très en profon-

\footnotetext{
1. On remarquera cependant que sur 830 occurrences de la base de données Dalloz, sur les risques naturels, depuis 2000 on ne trouve que 6 décisions concernant les submersions marines proprement dites. Le droit n'a donc pas pris la mesure complète de l'enjeu.

2. Le Parlement européen a adopté le 21 septembre 2010 une résolution sur la communication de la Commission intitulée : Une approche communautaire de la prévention des catastrophes naturelles ou d'origine humaine. Rappelant que «la prévention des catastrophes fait partie, au premier chef, des compétences des États membres et qu'il convient de continuer à tenir compte du principe de subsidiarité dans ce domaine », les députés européens insistent sur « la dimension et/ou la nature transfrontalière des catastrophes »; ce faisant, « il apparaît opportun et nécessaire de renforcer une coopération, tant au niveau régional qu'au niveau communautaire, fondée sur des actions complémentaires, sur la diffusion des meilleures pratiques et sur le principe de solidarité entre États membres ». Ils considèrent également que « la prévention devra constituer une étape de plus en plus importante dans le cycle de gestion des catastrophes ».
} 
deur. En effet, la plupart des dégâts matériels sont réparés sous un régime de responsabilité sans faute fondé soit sur le calcul actuariel des risques par les assurances, soit sur le principe de solidarité nationale inscrit dans notre Constitution (Conseil d'État, 2005). Cependant, le régime de la faute sous-tend les recours au juge par les victimes, ce qui aboutira souvent à une redéfinition du champ des risques devant être assurés et à une extension considérable des réparations qui mettent alors en jeu l'équilibre des systèmes de solidarité. Les véritables enjeux de la réparation sont bien ceux de la solidarité et de la responsabilité.

\section{La solidarité devant les réparations}

Selon une première estimation stabilisée en avril 2010 et qui a servi de critère à l'intervention du Fonds de solidarité de l'Union européenne (FSUE) ${ }^{3}$ la totalité des dégâts pour les 21 départements touchés s'élèverait à 2,379 Mds€ soit un milliard de moins que le seuil d'intervention du FSUE ${ }^{4}$ sur les catastrophes majeures. Cependant, dans le périmètre d'intervention régionale du FSUE, que l'on peut considérer comme le cœur des dégâts et qui concerne 10 communes de Vendée et 36 communes de Charente-Maritime, la mission, qui applique un coefficient multiplicateur de 1,20 pour tenir compte de l'expérience de la tempête Klauss de 2009, évalue les dégâts non assurés à $605 \mathrm{Mds} €$ et les dégâts assurés à 780 Mds€ soit un total de 1425,43 M€. Selon la Caisse Centrale de Réassurance ${ }^{5}$, les

3. Le seuil de 3,4 Mds€ nécessaire pour caractériser une catastrophe majeure ne sera pas atteint et il convient de solliciter la mobilisation du Fond de Solidarité de l’Union Européenne dans le cadre d'une procédure « régionale».

4. Selon le rapport de la mission interministérielle de juin 2010, intitulé : L'évaluation des dommages causés par la tempête Xynthia des 27 et 28 février 2010 à prendre en compte a titre du fonds de solidarité de l'union européenne : «Les critères cumulatifs d'éligibilité sont extrêmement contraignants, puisqu'ils impliquent, [...] que la majorité de la population de la région touchée soit affectée durablement dans ses conditions de vie et sa stabilité économique. En outre, le périmètre retenu doit être continu, à l'exception de la prise en compte de l'insularité. Ces contraintes [... impliquaient que la mission définisse, [...] un périmètre restreint regroupant les zones les plus affectées par la catastrophe, en tenant moins compte de l'ampleur des dégâts en valeur absolue, que de leurs effets relatifs sur les conditions de vie et la situation économique de la population. [...] Par ailleurs, la nécessité d'établir le caractère durable des dommages a conduit à retenir un périmètre privilégiant les effets de la submersion marine, qui provoque des dégradations de longue durée tant dans les habitations que dans les sols, plutôt que ceux du vent, plus rapidement réparables. »

5. Société privée à capital d'État remplaçant un ancien établissement public chargé de réassurer les sociétés d'assurances avec notamment des fonds garantis par l'État. La CCR a été créée par l'article 4 de la loi de 1982 : « La caisse centrale de réassurance est habilitée à pratiquer les opérations fonds propres des assurances et de la réassurance pourront facilement couvrir le besoin. Par ailleurs les fonds exceptionnels qui sont très nombreux, pourront couvrir les dommages non assurés des collectivités locales, des particuliers et agriculteurs, des pêcheurs, conchyliculteurs, PME, PMI, etc. Il faut rappeler que le fond CatNat qui est alimenté, comme le fond Barnier, par une surprime de $12 \%$ des contrats d'assurance habitation et $6 \%$ pour les véhicules, assure essentiellement les dégâts d'inondation et de submersion alors que les contrats de droit commun remboursent mieux les dommages dus au vent et à « la mouille»(Anziani, 2010). Le « fonds Barnier » quant à lui ${ }^{6}$, est attendu pour les rachats d'immeubles détruits et les études et travaux du plan digue. Il faut donc distinguer, pour la clarté de l'analyse, entre la solidarité purement assurancielle et la solidarité nationale avant d'exposer les enjeux juridiques d'une réforme proposée de ces différents systèmes.

\section{L'enjeu de la solidarité assurancielle}

Comme il a été dit par les assureurs devant la mission parlementaire, c'est le régime de droit commun des assurances qui va couvrir $90 \%$ des dommages à travers la prise en charge des biens assurés par les contrats d'assurance habitation (Anziani, 2010). Les assurances mutualistes, qui ne couvrent que $10 \%$ du marché national mais dont on ne sait pas le taux de couverture réelle dans la zone la plus touchée, ont annoncé qu'elles allaient devoir augmenter leurs cotisations en $2011^{7}$ pour faire face à la charge. On ne connaît pas la position des autres compagnies mais ce fait nous révèle l'ampleur de la responsabilité solidaire des assurés civils en France. À quoi il faut ajouter que, selon la fédération française des sociétés d'assurance (FFSA), les contrats d'assurance étant peu coûteux dans notre pays - mais universels - une surprime appliquée dans les zones à risques serait très peu dissuasive, au contraire des

de réassurance des risques résultant des catastrophes naturelles, avec la garantie de l'État. »

6. Fond de Prévention des Risques naturels Majeurs créé par la loi sur l'environnement de 1995 et fortement élargi depuis par diverses lois quand à son usage et à son abondement. Le fond dispose de 150 millions d'euros dont la moitié est déjà gagée par la prévention dans d'autres zones à risques, des études en cours et la réparation des ouragans martiniquais. Ce qui est loin des 500 millions nécessaires selon le rapport sénatorial pour indemniser les victimes surtout si on y ajoute une contribution du fonds à la réhabilitation ou amélioration des digues.

7. 3,5\% sur les contrats habitation et $2,5 \%$ sur les contrats véhicules de la MAIF. 
systèmes britanniques ou l'exposition aux risques entraîne une prime d'assurance très élevée. Le rapport parlementaire relève, comme un leitmotiv, la faiblesse de la culture du risque dans notre pays ce qui, ajouté à cette dilution des coûts d'assurance, ne permet pas de faire du marché privé de l'assurance un outil efficace de prévention. Il constitue en outre un encouragement aux spéculations hasardeuses sur des biens dont la valeur est obérée par l'omniprésence du risque. Le premier effet de la déclaration de catastrophe naturelle intervenue dès le lendemain de l'événement a été de donner aux assureurs l'accès à la réassurance par la Caisse Centrale de Réassurance (CCR) sur des fonds garantis par l'État même si cette demande de garantie n'était pas nécessaire pour cette fois aux yeux des fédérations d'assureurs.

Doit-on être rassuré par ces constatations? Il est curieux que le rapport parlementaire, qui passe beaucoup de temps à l'étude de la réforme du système d'indemnisation des catastrophes naturelles, ne se pose jamais la question de la justice de ces mécanismes de solidarité automatique qui permettent les comportements les plus irresponsables. Rappelons que les $4 / 5^{\mathrm{e}}$ des maisons détruites à La Faute-sur-Mer et L'Aiguillon-sur-Mer étaient des résidences secondaires, soit des biens plus voués aux loisirs et à la spéculation sur l'avenir qu'au besoin primaire de logements.

D'un point de vue prospectiviste, si les catastrophes d'ampleur régionale continuent de se produire avec la fréquence constatée dans les trente dernières années, chacune d'entre elles sera surmontable par les assureurs. Mais du fait de la répétition des sinistres, l'appel en garantie de l'État finira par devenir habituel, les réserves disponibles sur CatNat s'épuisant, et les fonds de solidarité comme le fonds Barnier étant rapidement asséchés.

Ce premier enjeu financier introduit un enjeu juridique de réforme des contrats en zone de risque, ce que le rapport envisage d'ailleurs très sérieusement dans ses propositions. Il se réfère, sans trop les approfondir, aux travaux récents inspirés par des catastrophes précédentes et dont les conclusions étaient moins optimistes (Bentoglio et Betbèze, 2004).

En 2004, par exemple, l'exercice prospectif du Commissariat général au Plan soulignait, d'une part, le rôle clef de l'assurance pour l'entreprise et l'acti- vité sociale et, d'autre part, le rôle de l'État pour les risques non mutualisables et les sinistres majeurs, alors que le risque pourra s'aggraver pour deux raisons. Premièrement, la demande de protection peut entraîner la dérive de l'assurance et de ses coûts et, deuxièmement, la concentration des richesses et des activités dans des zones soumises à une augmentation des aléas pourraient faire exploser le système assuranciel français ${ }^{8}$.

La pression des victimes, l'élargissement du domaine des risques assurables et les primes insuffisantes constituent les enjeux juridiques qui découlent des enjeux sociaux et économiques. À l'État d’y répondre par un aménagement du territoire plus soucieux de l'évaluation des coûts de catastrophes et par un mécanisme de bonus/malus sur l'intervention des systèmes d'indemnisation de l'État lorsque les assurés se sont installés dans des zones à risque. Sans ignorer la difficulté de tels mécanismes ni la solidarité nationale ni le fait que la sanction financière et le dispositif assuranciel ne peuvent garantir des comportements plus prudents, le groupe soutient l'idée que, pour pérenniser le système CatNat, il faut trouver un équilibre entre la responsabilité individuelle et la solidarité nationale grâce à des mécanismes d'incitation à la prévention fondés sur une bonne connaissance des risques. Se contenter de la réponse rassurante quant aux capacités d'indemnisation des dommages de Xynthia et à la faiblesse d'une incitation par les primes reviendrait à ignorer l'enjeu de justice sociale et de conservation économique à plus long terme des zones à risques que décrivait ce premier rapport.

Le second rapport, rédigé par les inspections générales de trois ministères ayant mené la « Mission d'enquête sur le régime d'indemnisation des victimes de catastrophes naturelles en 2005 » relève que « la contribution des assureurs à la politique de prévention n'est pas à la hauteur de celle d'autres pays ». Il est certain que les législations relatives à la prévention et à l'indemnisation des risques de catastrophes naturelles sont juxtaposées, mal coordonnées et mal appliquées. Les premières mettent l'accent sur la protection des vies humaines sans

\footnotetext{
8. Le rapport souligne alors «l'amplification de risques extrêmes. Ces risques, déjà connus (catastrophes naturelles, explosions industrielles...) sont susceptibles de se multiplier dans la décennie à venir, sous l'effet de la concentration géographique des biens et des personnes, de l'interdépendance accrue des échanges et des activités, ainsi que de changements de contexte comme le réchauffement climatique, s’il se confirme».
} 
trop se préoccuper les risques économiques qui sont au cœur des secondes. Ainsi les assureurs sont peu impliqués dans la prévention et les « sanctions » par la non-indemnisation dans les zones des Plans de Prévention des Risques sont contournées par l'intervention des fonds de solidarité.

C'est donc à juste titre que le Conseil d'État a rappelé en 2004 que " pour être viable, la socialisation du risque doit aller de pair avec la prévention des risques ».

\section{Les enjeux juridiques liés à la réparation par la solidarité nationale}

Bien avant l'inscription, par la constitution de 1946, du droit créance des citoyens envers la solidarité nationale, Maurice Hauriou dans ses conclusions sous l'arrêt Cames en décrivait parfaitement les justifications ${ }^{9}$. La responsabilité sans faute de l'État joue pleinement même si la cause matérielle du dommage reste inconnue et l'on connaît le succès de cette idée qui a inspiré toute l'évolution du concept de solidarité nationale au long du $\mathrm{Xx}^{\mathrm{e}}$ siècle (Conseil d'État, 2005).

\section{La multiplication des dispositifs de solidarité nuit à leur efficacité}

Ici encore, Xynthia est un révélateur des défauts du système. L'accumulation des fonds de solidarité, chacun dévolu à une catégorie de victimes, pose des problèmes de frontières entre dommages réparables et crée un sentiment de garantie absolue qui déresponsabilise les personnes exposées ${ }^{10}$. D’autres intérêts économiques que ceux des habitants feront donc intervenir des fonds spécialisés ${ }^{11}$. La coordina-

9. «Les choses se passent comme si l'État, en sa qualité de personne morale, gérait une assurance mutuelle contractée entre les administrés contre le risque des accidents administratifs. L'idée d'une assurance pareille procède logiquement du principe d'égalité devant les charges publiques. $[\ldots]$ et les indemnités compensatrices peuvent très bien être considérées comme versées par une sorte de caisse mutuelle associée aux services publics et alimentée par une prime incorporée à l'impôt... L'égalité devant les charges publiques doit être poursuivie directement là où il est possible de la réaliser, par exemple, en matière d'impôt ou de service militaire; elle doit l'être indirectement par la voie d'indemnités compensatrices dans le cas où la charge publique ou le fait de l'administration sont d'une nature telle que par eux-mêmes ils retombent inégalement sur les administrés. » CE, 21 juin 1895, Cames : conclusions Hauriou, Rec. CE 1895, 509 p.

10. Rappelons que le régime légal d'assurance des catastrophes naturelles, dit «Cat Nat », issu de la loi n 82-600 du 13 juillet 1982 porte sur « les dommages matériels directs non assurables ayant eu pour cause déterminante l'intensité anormale d'un agent naturel, lorsque les mesures habituelles à prendre pour prévenir ces dommages n'ont pu empêcher leur survenance ou n'ont pu être prises l'article (L. 125-1 du code des assurances).

11. Fonds national de garantie des calamités agricoles (FNGCA). Dans les deux départements les plus touchés, 50000 ha et 1000 exploitations tion des interventions pour une couverture optimale des dommages est donc un enjeu important dont on voit mal la mise en œuvre pour le moment.

\section{Vers un dévoiement du Fonds Barnier?}

En proposant que le Fonds de prévention des risques naturels majeurs (FPRNM) finance le rachat des maisons détruites en zones noires dites désormais "zones de solidarité ", la mission met en cause l'esprit même de ce fonds de prévention et pas seulement son équilibre financier. Créé en 1995, ce fonds doit préserver les vies humaines par des actions de prévention dont l'évacuation des zones les plus dangereuses grâce à des expropriations. Or on propose ici d'utiliser au moins $50 \%$ des crédits du fonds, soit 75 millions/an, pour financer les 500 millions nécessaires au rachat des maisons détruites. En rebaptisant zone de solidarité ce qui fut très justement une zone noire on effectue un tour de passe-passe sémantique qui permet ce dévoiement du fonds Barnier qui

agricoles, auxquelles il faut ajouter autant d'exploitations conchylicoles ont subi des pertes matérielles et de potentiel agronomique du fait notamment de la stagnation des eaux salées restée plus de trois jours derrière les digues. Les aides pour indemniser les calamités agricoles doivent obtenir le feu vert de la commission européenne et la procédure d'attribution semble compliquée et lente. Aux $1000 €$ par ha indemnisés s'ajoutent $30000 €$ par exploitation au titre du fonds d'allégement des charges (FAC).

Fonds d'intervention pour les services, l'artisanat et le commerce (FISAC). C'est un dispositif fondé sur la solidarité financière entre la grande distribution et les petites entreprises commerciales et artisanales. Il est alimenté par un prélèvement sur l'excédent du produit de la taxe d'aide au commerce et à l'artisanat (TACA) acquittée par la grande distribution. La TASCOM (Taxe sur les surfaces commerciales) est désormais affectée au budget général de l'État et attribue des subventions variant de $20 \%$ minimum pour les dépenses d'investissement matériel à $50 \%$ maximum des dépenses de fonctionnement et d'investissement immatériel à toute une série de bénéficiaires : maîtres d’ouvrage publics (communes, groupements de communes ou associations, établissements publics), entreprises et groupements d'entreprises ou coopératives. Les collectivités locales, pour voir leurs dommages non assurables ou les dépenses supplémentaires engagées du fait de la catastrophe, peuvent être aidées par l'État selon deux voies : le «fonds de solidarité en faveur des collectivités territoriales et de leurs groupements touchés par des catastrophes naturelles » qui concerne les dommages les moins importants (entre 150000 euros et 4 millions d'euros) et la «subvention d'équipement aux collectivités locales pour la réparation des dégâts causés par les calamités publiques » alimentée par une loi de finances rectificatives ou un décret d'avance. Dans le même ordre d'idée il faudra compenser les pertes fiscales des collectivités qui s'élèvent à 1,8 million d'euros, pour la disparition de 1510 habitations.

L'union européenne est impliquée à la fois par son fonds d'urgence dit «mesure 126 », et par le Fonds de Solidarité de l'UE qui n’intervient comme il a été dit qu’à partir de 3,5 milliards d'euros de dégâts estimés. Comme les dégâts de la tempête dans le périmètre central déclaré à la Commission européenne ne s'élèvent qu'à 2,5 milliards, les aides seront calculées dans la catégorie des événements régionaux. Tout se passe comme si on devait distinguer entre une solidarité européenne réservée à des événements énormes et une solidarité nationale pour des faits exceptionnels mais gérables par l'État en cause. On peut aussi espérer une réaffectation des fonds européens FEDER, FSE, déjà engagée pour le FEDER en Région Poitou-Charentes. 
n'aura jamais les moyens de cette nouvelle mission même au prix d'une augmentation des primes d'assurance. Si le Fonds est déjà intervenu dans l'urgence pour amorcer le courant de la solidarité nationale vers quelques victimes, on ne doit pas s'attendre à un règlement rapide de tous les cas à moins d'un investissement massif de l'État. Et on ne peut qu'être choqué de cet appel au Fonds Barnier alors que les sommes rendues disponibles par les assureurs s'élèvent à 1,3 milliard d'euros! De plus, est-ce à la seule solidarité automatique des assurés français de financer tous les engagements de la solidarité nationale alors que l'État, les acteurs locaux et le marché immobilier ont une part certaine de responsabilité dans la catastrophe? Doit-on mettre en péril les moyens de la prévention des événements futurs en consacrant l'idée que la réparation est plus importante que les plans et les travaux de prévention? Et cela au moment où la loi dite Grenelle II ${ }^{12}$ intègre la directive inondation en donnant aux plans de prévention une force obligatoire qu'ils n'avaient pas jusqu'ici ${ }^{13}$. Peut-on croire que de telles annonces pourront éviter quelques contentieux retentissants initiés par des victimes toujours insatisfaites de ces efforts?

\section{La responsabilité devant les réparations}

On peut, en effet, s'attendre à ce que la fonction cathartique et psychothérapique du procès pénal joue à plein dans les années qui viennent (Cimelière, 2010; Huglo, 2010). En outre, les dommages matériels sont considérables et la lenteur des réparations ou compensations amiables ne pourra qu'inciter les victimes à rechercher le règlement judiciaire (Jeanneaux et Sabau, 2000), ne serait-ce qu'à titre de précaution avec une prédilection pour le juge pénal estimé plus efficace pour obtenir réparation (Danet, 2004, 2006). Cependant le juge administratif pourrait être fortement sollicité en raison de

12. Loi portant Engagement National pour l'Environnement 2010-788 du 12 juillet 2010, publiée au JO du 13 juillet.

13. Directive 2007/60/CE du Parlement européen et du Conseil du 23 octobre 2007 relative à l'évaluation et à la gestion des risques d'inondation. Article L 566-1 du code de l'environnement. La stratégie nationale de gestion des risques permettra d'identifier les territoires menacés notamment par les submersions marines par une coopération entre État et collectivités locales à l'échelon du Bassin. Le tout se décline en plans de gestion des risques et en stratégies locales coordonnées par l'autorité de bassin et commandant la réalisation des PPRN. SCOT et PLU doivent alors être mis en compatibilité avec le plan de gestion des risques d'inondations. l'implication défaillante de la puissance publique dans la prévention comme dans les ouvrages.

Les limites de la mise en cause pénale des responsables publics

Pour de multiples raisons plus sociologiques que juridiques ${ }^{14}$, les victimes s'adresseront de préférence au juge pénal ${ }^{15}$, ce qui suppose de porter plainte avec constitution de partie civile contre le responsable. Le système français interdit à un procureur de classer la plainte lorsqu'il y a constitution de partie civile et la loi du 15 juin 2000 a facilité l'accès des victimes à l'information sur leurs droits et l'intervention au procès (Agostini, 2000). Il y faut cependant un préjudice personnel et directement lié à l'infraction, le tout dans des limites de procédures assez strictes. L'ouverture législative de l'action civile aux associations d'environnement et aux associations de victimes devrait faciliter les constitutions collectives face à un événement aussi destructeur ${ }^{16}$. Rappelons que l'accès au procès ne crée pas automatiquement un droit à réparation ${ }^{17}$. Cela dit, deux sources de procès peuvent être activées.

Pour une faute prenant son origine dans les travaux ou le mauvais entretien des ouvrages publics et parce qu'il s'agit d'une matière susceptible de délégation de service public, les personnes morales que sont les collectivités locales mais aussi les associations de propriétaires chargées des digues pourraient être l'objet de poursuites au titre de l'article 121-2 du code pénal ${ }^{18}$.

14. Christian Huglo se pose la question des raisons de l'attrait pour le procès pénal? La réforme du Code pénal de 1994, qui a institué une responsabilité des personnes morales en matière d'environnement, le recours à l'expertise gratuite, l'ouverture du procès pénal aux associations de défense de l'environnement, aux associations de victimes et même aux collectivités publiques pour tous les dommages directs ou indirects, en sont les trois raisons selon lui.

15. Depuis l'arrêt Laurent-Atthalin (Crim. 8 décembre 1906, Bull. $n^{\circ} 443$ ) les pouvoirs publics sont légalement obligés à engager les poursuites judiciaires lorsque le plaignant s'est constitué partie civile.

16. Protection de l'environnement (articles 2-13 du Code pénal, L. 252-1 et suivants, L. 253-1 du Code rural...) Atteintes aux personnes (articles 2-2, 2-3, 2-9, 2-12, 2-15, 2-16 du Code pénal).

17. Selon $\mathrm{M}^{\mathrm{me}}$ Agostini : La recevabilité de l'action, qui l'autorise à participer au procès pénal, n'a pas pour conséquence inéluctable la recevabilité puis le bien-fondé de la demande en réparation (Crim. 19 sept. 2000, Bull. $\left.n^{\circ} 271\right)$.

18. Article 121-2 : Les personnes morales, à l'exclusion de l'État, sont responsables pénalement, selon les distinctions des articles 121-4 à 121-7 des infractions commises, pour leur compte, par leurs organes ou représentants. Toutefois, les collectivités territoriales et leurs groupements ne sont responsables pénalement que des infractions commises dans l'exercice d'activités susceptibles de faire l'objet de conventions de délégation de service public. La responsabilité pénale des personnes morales n'exclut pas celle des personnes physiques auteurs ou complices 
En revanche pour des fautes relevant de la prévention, de l'alerte, de la protection et des secours aux populations avant ou pendant l'événement, les personnes morales ne peuvent être mises en cause. Ce sont donc des personnes physiques, représentantes des autorités responsables, qui peuvent être poursuivies pour mise en danger d'autrui, selon l'article 121-3 ${ }^{19}$.

Cependant, mise en cause et condamnations dépendront à la fois des droits de la partie civile au procès pénal et de l'appréciation in concreto des fautes prétendues et de leur lien direct avec le dommage subi par les plaignants.

Le maire peut être pénalement responsable du fait de l'exercice de ses pouvoirs de police, lorsqu'il ne respecte pas l'obligation de sécurité, d'information préalable et d'assistance que la loi lui impose en matière de prévention des risques naturels. Bien évidemment le préfet exerçant cette autorité de police générale ou spéciale sur son département peut se retrouver dans la même situation.

À ce cas général on doit ajouter les incriminations du fait du non respect des règles d'urbanisme. Ainsi, délivrer une autorisation en toute méconnaissance d'un PPR approuvé engage la responsabilité sauf à considérer que les conditions imposées à la construction prenaient déjà le risque en compte et de manière suffisante ${ }^{20}$. Mais c'est l'appréciation du risque réel qui importe et l'article R. 111-2 permet au maire ou au préfet de s'opposer à une construction ou imposer des prescriptions spéciales en cas d'atteinte à la salubrité ou à la sécurité publique. La décision Bethon du Tribunal Administratif de

des mêmes faits, sous réserve des dispositions du quatrième alinéa de l'article 121-3.

19. Article 121-3: Il n’y a point de crime ou de délit sans intention de le commettre.

Toutefois, lorsque la loi le prévoit, il y a délit en cas de mise en danger délibérée de la personne d'autrui.

Il y a également délit, lorsque la loi le prévoit, en cas de faute d'imprudence, de négligence ou de manquement à une obligation de prudence ou de sécurité prévue par la loi ou le règlement, s'il est établi que l'auteur des faits n'a pas accompli les diligences normales compte tenu, le cas échéant, de la nature de ses missions ou de ses fonctions, de ses compétences ainsi que du pouvoir et des moyens dont il disposait.

Dans le cas prévu par l'alinéa qui précède, les personnes physiques qui n'ont pas causé directement le dommage, mais qui ont créé ou contribué à créer la situation qui a permis la réalisation du dommage ou qui n'ont pas pris les mesures permettant de l'éviter, sont responsables pénalement s’il est établi qu'elles ont, soit violé de façon manifestement délibérée une obligation particulière de prudence ou de sécurité prévue par la loi ou le règlement, soit commis une faute caractérisée et qui exposait autrui à un risque d'une particulière gravité qu'elles ne pouvaient ignorer. Il n'y a point de contravention en cas de force majeure.

20. CE, 23 déc. 1994, Min. Équipement c. M. Y... Lebon Tables, $n^{\circ} 108969$.
Nantes ${ }^{21}$ illustre parfaitement ce cas de déféré préfectoral, au titre de R. 111-2 contre un permis accordé derrière la digue du Lay.

Quant à l'infraction fondée sur l'article 223-1 du Code pénal qui définit le délit de mise en danger d'autrui ${ }^{22}$, elle sera toujours difficile à démontrer car elle doit nécessairement constituer la violation d'une obligation imposée par la loi ou les règlements, comporter l'intention délibérer de violer une obligation de sécurité ou de prudence ou la commission flagrante d'une imprudence. C'est donc très largement la connaissance du risque qui est à la base du système. Enfin, la faute pénale des personnes morales chargées de l'entretien et de la surveillance des digues pourrait être retenue sur les mêmes fondements de 121-2 et 223-1, sans préjudice d'une action civile.

\section{La responsabilité administrative au coeur des réparations}

Les victimes pourront demander, à l'État et aux collectivités locales, réparation des dommages matériels et du pretium doloris. Rappelons simplement que deux régimes sont en cause :

- celui de la responsabilité sans faute du fait de la mauvaise conception ou du mauvais entretien des ouvrages publics. Dans la mesure où communes, État et département auront pris en charge financièrement et techniquement les travaux sur les digues, leur implication est possible sur ce motif;

- celui de la responsabilité pour faute de la police administrative de la sécurité ${ }^{23}$. Les retards dans l'ap-

21. TA de Nantes 30 mars 2010 M et Mme Bethon Nº81045. Décision analysée infra en seconde partie.

22. Article 223-1 : « Le fait d'exposer directement autrui à un risque immédiat de mort ou de blessure de nature à entraîner une mutilation ou une infirmité permanente par la violation manifestement délibérée d'une obligation particulière de sécurité ou de prudence imposée par la loi ou le règlement. »

23. Le professeur J.-M. Pontier (2003) résume cette responsabilité en quelques mots : Ainsi, une commune ne saurait s'exonérer de la responsabilité qu'elle a encourue "dans l'exercice de la mission de prévention des inondations qui lui incombe” en invoquant les fautes qu'aurait commises le service d'annonce des crues mis en place par l'État en tardant à informer les services municipaux de la montée des eaux (CE 22 juin 1987, Ville de Rennes c/ Compagnie rennaise du linoléum et du caoutchouc, Lebon p. 223, AJDA, 1988, p. 65, note J. Moreau). De même, en ne prenant aucune mesure «propre à avertir les plaisanciers » du danger exceptionnel constitué par la présence d'une ligne à haute tension, fût-elle à hauteur réglementaire, à proximité d'une rampe d'accès des voiliers à un lac, le maire a commis dans l'exercice de ses pouvoirs de police une faute de nature à engager la responsabilité de la collectivité (CE 8 novembre 1985, Rijlaarsdam, Lebon tables, p. 523). Plus généralement, l'absence de mesures de police qui auraient pu empêcher la survenance d'un dommage est une faute engageant la responsabilité de la collectivité (par exemple, CE 14 mai 1986, Commune de Cilaos, Lebon p. 426). 
plication des PPR, les zonages urbains établis en méconnaissance complète des risques, les autorisations individuelles accordées sans tenir compte de la réalité de l'exposition au danger peuvent en effet constituer de telles fautes.

Le contrôle du juge sur l'erreur manifeste d'appréciation que constitue l'ignorance d'une étude ou d'un avertissement transmis par le préfet jouera pleinement pour établir cette responsabilité. Le service auquel l'autorité du permis de construire délègue son instruction et qui ne préviens pas du danger en conseillant de refuser le permis sera conjointement impliqué dans le partage de la responsabilité. Il faut bien entendu que les risques soient connus de l'administration à la date de la délivrance des autorisations. Mais n'est-ce pas le cas ici pour toutes les constructions récentes?

On se posera certainement la question de savoir si les digues de Charente et de Vendée avaient pour but de garantir les terres agricoles de cette vieille région de poldérisation ou de protéger les zones urbaines qui se sont implantées derrière sans que la question de leur adaptation au risque nouveau soit posée? On se posera encore la question de l'imprévisibilité d'un tel événement dont l'ampleur fut exceptionnelle et il y sera répondu en considérant non seulement la force des éléments, que le juge qualifie le plus souvent de prévisibles, mais aussi les nombreux avertissements donnés dans les années précédentes et qui rendaient la probabilité très forte. Le fait même de l'étude des PPR suffira à démontrer la prévisibilité du danger.

À l'inverse l'obligation de prudence, et donc la faute, concerne aussi les victimes car s'installer sans précautions particulières, dans une architecture inadaptée à la cinétique de la submersion marine, peut constituer une telle imprudence à condition bien entendu que des solutions plus efficaces n'aient pas été interdites par le règlement de construction applicable ${ }^{24}$.

24. CE, 27 novembre 1987, Société Provençale d'équipement, RFDA, 1987, p. 384 : «Cons. en revanche qu'il résulte de l'instruction que la société Comptoir des nouveautés mondiales et la société de Mécanique provençale ne pouvaient ignorer ni les risques naturels d'inondation des lieux qui existaient au moment où elles ont pris la décision de s'installer sur la zone industrielle ni les risques qui subsistaient après l'achèvement des travaux d'aménagement et sur lesquels elles avaient elles-mêmes attiré l'attention du concessionnaire; qu'elles n'ont pris néanmoins aucune mesure de précaution tendant notamment à surélever les sols du bâtiment et dont la nécessité, rappelée par l'expert commis par le tribunal administratif, n'est pas contestée; que l'imprudence ainsi commise par les victimes est de nature à atténuer la responsabilité des auteurs du
Enfin et sans pouvoir en donner le détail, nous disposons d'une abondante jurisprudence du contentieux des catastrophes naturelles qui signale l'enjeu de l'évaluation des dommages et des causes d'exonération partielle ou totale de l'assureur. Cette branche du contentieux devrait aussi se développer dans les zones de Xynthia même si, dans l'urgence, les assureurs sont réputés avoir vite et très libéralement fait le nécessaire.

\section{LES ENJEUX DE LA PRÉVENTION}

Contrairement à la réparation qui fait le décompte présent des morts et des destructions, le discours sur la prévention est toujours à cheval entre le passé et l'avenir. Il s'agit en effet de critiquer les défauts de la prévention organisée avant la catastrophe et d'imaginer une prévention idéale pour l'avenir. La proposition peut alors se heurter à la volonté quasiment thérapeutique des victimes d'oublier le pire et de le rendre impensable, donc non reproductible dans l'avenir. Une organisation institutionnelle de la participation des citoyens à la prévention d'autres tempêtes et submersion apparaît comme un remède et un enjeu essentiels de l'action des pouvoirs publics puisque ces derniers sont, mieux que le juge et les assureurs, au nœud de la problématique.

Depuis 1982 la prévention est bien le complément nécessaire de la solidarité. Le système de 1987 s'est donc enrichi de réformes successives en 1995, 2003 puis 2010. Certes, les textes paraissent ignorer la submersion marine ${ }^{25}$. Mais l'ensemble du dispositif ordinaire aurait dû permettre de prévenir et de circonscrire un risque évident, même si on ne pouvait en connaître les formes et l'amplitude.

La prévention par la contrainte de police n'a donc pas fonctionné de manière satisfaisante. Deux autres formes de prévention ont été trop négligées, la prévention par les mesures opérationnelles, foncières et techniques et la prévention par la participation des citoyens grâce à une véritable gouvernance des territoires du risque. Les enjeux de l'après Xynthia

dommage; qu'en fixant au 2/5 la part des conséquences dommageables des inondations qui doit être laissée à la charge des victimes, le tribunal administratif a fait une juste appréciation des circonstances de l'espèce. »

25. La submersion marine n'apparaît que dans une circulaire relative aux catastrophes naturelles. Circ. Interministérielle Intérieur Économie du 19 mai 1998, complétée par la circ. du 24 novembre 2000 (NOR : INTE 0000267C). Elle vient d'être intégrée par la loi Grenelle II dans les dispositions relatives aux inondations. 
portent donc sur l'effectivité de la première prévention et sur la mise en œuvre des deux autres.

\section{La prévention par la contrainte}

La contrainte peut intervenir à deux moments, celui de la planification des risques, simultanément de la planification urbaine et celui de la mesure individuelle, lorsque des permis sont sollicités en zone de risque et alors même que le zonage urbain permettrait la construction.

La prévention par une meilleure coordination entre documents d'urbanisme et servitudes d'utilité publique

La rente immobilière est au cœur de la relation entre PLU et PPRN. Les documents d'urbanisme sont des documents à vocation générale (Dieu, 2007) rendus très complexes par le croisement d'objectifs d'aménagement du territoire qui peuvent se contredire. Avec la loi SRU la séparation des fonctions est acquise entre des documents à vocation générale indiquant les orientations de l'aménagement : SCOT, SMVM, DTA, etc. ${ }^{26}$, et les documents locaux, à l'échelle d'une commune ou d'une intercommunalité, qui appliquent les règles supérieures et les orientations d'aménagement tout en organisant la répartition des droits de construire. Réalisé par le conseil municipal ou l'assemblée intercommunale, le plan local d'urbanisme (PLU) pousserait-il les élus à la schizophrénie? Il doit en effet garantir les protections nécessaires de l'environnement, de la forêt et de l'agriculture, ainsi que la prise en compte des risques naturels et technologiques, tout en déterminant pour chaque parcelle l'étendue des droits à construire qui deviennent un élément réel de la propriété et fixent donc la valeur de nombreux patrimoines familiaux. Le classement en zone constructible d'une propriété se faisant sous le regard des électeurs de la commune, on comprend l'importance politique du débat qui finit toujours par minimiser les risques naturels ou par les nier au motif de la présence d'une digue protectrice. Exigence d'autant plus impérative que la demande de terrains à bâtir augmente sur un espace

26. Schéma de cohérence territoriale à l'échelle de l'agglomération, éventuellement couplé avec un schéma de mise en valeur de la mer, Directive territoriale d'aménagement et Directive territoriale d'aménagement et de développement durable du territoire instituée par la loi Grenelle II. littoral étroit et de plus en plus rare. La rente foncière littorale est en constante progression et le PLU devient, aux yeux du public et de nombreux élus, presque essentiellement une machine à distribuer la plus value foncière. Ni la qualité des paysages et de l'environnement naturel, ni la valeur agronomique, souvent excellente, des sols cultivés, ni la crainte de la nature, ne peuvent moraliser ce marché qui se nourrit de sa propre rareté. Seules des règles légales défendant ces intérêts généraux, sans ambiguïté et à condition d'être appliquées avec sévérité par l'administration ${ }^{27}$, peuvent bloquer ou ralentir l'urbanisation littorale. Ainsi, quand il s'agit de ne pas exposer davantage de population à un risque naturel, la rente foncière joue à plein contre la sécurité en freinant une application rigoureuse de la loi.

Car les servitudes imposées par le plan de prévention des risques naturels qui a remplacé les divers plans de prévention des risques d'inondation (PPRI) applicables avant 1995, exercent l'effet inverse des PLU sur la rente foncière en l'annulant ou en la désintéressant. Le plan de prévention des risques naturels détermine une "zone exposée aux risques $^{28}$ » dans laquelle sont interdits tous types de construction. Cependant le préfet peut y autoriser des installations de type économique pour les exploitations agricoles, forestières et industrielles avec des prescriptions visant à ne pas aggraver les risques pour des vies humaines. Le même article L. 562-1 détermine les zones qui ne sont pas directement exposées aux risques mais où des installations pourraient aggraver ceux-ci, notamment pour les habitants et, dans lesquelles, des prescriptions ou des interdictions peuvent être fixées par le Préfet.

On se rappellera que le risque est constitué de trois éléments, l'aléa naturel plus l'intensité de l'exposition des biens et des personnes plus la cinétique de l'événement. Ce qui donne tout son sens à un véritable élargissement des possibilités de prescription données au préfet par la loi Grenelle II. Il interdira la construction de logements dans la première

27. Entre 1982 et 2001, 35923 communes françaises ont subi au total 110823 catastrophes naturelles. Ces dernières ont fait l'objet de 247 arrêtés ministériels reconnaissant ces événements dans les communes concernées et permettant aux victimes d'être indemnisées par leurs assurances. Actuellement, selon l'IFEN, près de 9000 PPRN sont en place. La plupart concernent les inondations $(71 \%)$ et les mouvements de terrain (21\%). 1999-2000 est l'année de rupture dans la progression des PPRN.

28. Selon la nouvelle rédaction de l'article L 562-1 du Code de l'Environnement, par la loi ENE de 2010, les termes de zone rouge et de zone de précaution ont disparu. 
zone et pourra l'interdire ou y mettre des conditions drastiques dans la seconde. Il pourra aussi contrôler les installations économiques pour éviter les dommages trop importants dans la première et pour éviter qu'elles aggravent la situation de l'habitat dans la seconde. Ainsi le Plan modifiera le droit réel de chaque parcelle pour l'adapter à la réalité du risque. Il n'y a plus de distinction entre la zone de précaution et la zone de danger mais une réponse obligatoirement adéquate à un danger omniprésent dont l'intensité, variable dans l'espace, commande ces réponses. Ainsi le plan de prévention ne peut qu'amputer, parfois considérablement, la valeur foncière des biens concernés et limiter, sinon mettre en péril la plus-value de la rente foncière littorale. On comprend alors pourquoi les préfets ont tant de difficultés à imposer les PPR et pourquoi les PLU intègrent aussi mal leurs dispositions, comme le relève le rapport d'enquête parlementaire ${ }^{29}$. Les plans communaux de sauvegarde sont quasiment inexistants dans les zones les plus exposées et, de l'aveu même du Ministère (MEEDDM) auditionné par la Mission sénatoriale, la concertation avec les collectivités en cause est très défectueuse. Enfin, selon la Direction de la Sécurité civile, seules 1474 des 7660 communes soumises à cette obligation légale l'avaient satisfaite en 2009. Souvent très légers lorsqu'ils existent, seuls 14 de ces plans ont été approuvés sur les 69 communes de Vendée qui auraient dues en être pourvues.

Réécriture du rapport entre l'impératif vital et la rente par la loi Grenelle II

Au moment de la tempête, avec une précision cruelle, la Mission sénatoriale souligne pêle-mêle l'inadaptation par obsolescence des documents d'urbanisme à la prévention des risques, le nombre important de constructions illégales, phénomène ancien et récurrent dans les espaces proches de la mer, comme les lotissements construits dans les zones manifestement dangereuses, "les risques naturels n'ayant pas été ignorés mais négligés ».

Il nous faut bien parler d'impuissance de l'État qui, depuis des décennies ne sait protéger ni les sites, ni les zones à risques sur le littoral lorsque

29. Le rapport du Sénat précité relève que 71 PPR ont été prescrits sur les 864 communes littorales exposées au risque de submersion marine alors que 800 de ces communes sont classées par la fédération française des sociétés d'assurance en zone submersible entre 0 et $2 \mathrm{~m}$ au-dessus du niveau de la mer soit 235000 maisons concernées! la pression y est forte et ancienne. Une obligation plus drastique d'intégrer le risque dans les PLU ne rétablirait-elle pas un meilleur équilibre entre les enjeux de la plus-value foncière et ceux de la protection des populations? Que le PLU soit contraint d'annexer le PPRN en tant que servitude d'utilité publique signifie bien l'obligation de l'appliquer strictement au zonage urbain. Mais cette cohérence n’a jamais été respectée pour au moins trois raisons. La première est que la corrélation dans le temps des deux documents n'était pas parfaitement organisée par la loi. Deuxièmement, le PLU ne dispose que pour des aménagements à venir sans pouvoir imposer des obligations de faire aux bâtiments existants dont les propriétaires ne demandent aucune transformation $^{30}$. Troisième raison enfin, la marge d'appréciation du risque par la commune est encore trop grande mais, pour autant, le PPRN n'est pas sans effet sur l'urbanisme local car il commande la décision sur les autorisations individuelles et il signale des risques dont la police municipale doit tenir compte sous peine d'une grande responsabilité du maire et de la commune en cas d'accident.

La mission parlementaire s'est fait expliquer le principe d'indépendance des législations et ses effets sur le rapport des PPR avec les PLU ${ }^{31}$. Selon l'article L. 564-2 du code de l'environnement, les PPR, qui ont valeur de servitude d'utilité publique, doivent être annexés au document d'urbanisme élaboré par la commune. Et la circulaire du $1^{\text {er }}$ septembre 2009 relative au contrôle de légalité recommande aux préfets de les invoquer à l'encontre des autorisations d'urbanisme données dans les zones de danger. Cependant, le déféré préfectoral ne fait

30. Voir le rapport de la mission interministérielle de juin 2010 précité, pages 41 et 49 : «En définitive, la mission fait le constat de sérieuses carences dans la mise en œuvre sur le plan local de la politique nationale de prévention des risques naturels, et cela aussi bien en matière de constitution et de mise à jour des atlas des zones inondables (AZI) y compris des quelques atlas existants en matière de submersion marine, que de réalisation des plans de prévention des risques inondation et submersion marine (PPR). » En outre, le Pr. J.-B. Auby a fait remarquer que nombre des maisons détruites par Xynthia avaient été construites avant que la loi réforme les PPR et en impose la mise en annexe des PLU.

31. Le rapport Anziani (2010) exprime ainsi le principe pour en dénoncer les effets : « [p. 68 du rapport] Au nom du principe d'indépendance des législations, seuls les documents, les servitudes et les règles prévus par le code de l'urbanisme sont pleinement opposables aux actes pris dans cette matière. Cette vision juridique provoque une distinction presque imperméable entre l'urbanisme et la gestion des risques naturels. Cloisonnés et segmentés, ces deux domaines ne communiquent entre eux que de manière ponctuelle et incomplète. Le doyen Jean-Bernard Auby soulignait que la dualité entre le code de l'environnement et le code de l'urbanisme avait donné naissance à une "pluralité excessive de documents" qui créaient des zonages concurrents, voire contradictoires, et qui étaient un facteur de confusion et d'incompréhension pour les usagers. » 
que relever l'illégalité du PLU dont l'absence de mise à jour constituera éventuellement une erreur manifeste d'appréciation. Elle ne contraint pas la commune à une révision, qui serait une mise à jour impérative à moins qu'elle soit imposée par le pré$\mathrm{fet}^{32}$. Ce dont on peut tirer deux enseignements : d'une part, la conformité du PLU au PPR n'est exigible que pour une révision postérieure à l'application, ou exigée par le préfet, anticipée ou non, du PPR et, d'autre part, le PPR est bien un document supérieur au PLU même si cette supériorité ne peut s'imposer que de façon indirecte.

Ainsi, le principe d'indépendance des législations a joué à plein contre une meilleure corrélation des documents. S'y ajoute la mauvaise volonté mise par des communes à accepter la publication du plan de prévention qui détruit la rente en grande partie. Mais il ne faut pas oublier qu'indépendance n'a jamais voulu dire cloisonnement étanche ni obstruction systématique d'un intérêt général par un autre. C'est pourquoi la Loi de 2010 apporte quelques correctifs importants pour l'avenir.

Les correctifs apportés en 2010 s'inscrivent dans une évolution de la Doctrine prise entre deux approches du problème qui ressortent clairement des auditions des professeurs J.-B. Auby et Y. Jégouzo par la Mission sénatoriale (Anziani, 2010). Soit pour stigmatiser la mauvaise application des lois par une administration qui ne sait pas imposer les PPR aux communes. Soit pour regretter la complexité d'un système qui fait du PLU le document de synthèse de tous les intérêts d'environnement, de sécurité et de développement territorial en le chargeant de résoudre la complexité d'une accumulation considérable d'outils qui ne sont pas de la même valeur et ne poursuivent pas le même objectif. C'est peut-être pour consacrer cette supériorité du PPR, mal appliquée par l'administration, que le juge continue d'affirmer une position dissidente et très contestée par la Doctrine ${ }^{33}$ sur la qualification

32. Ce qu'illustre très bien le jugement du TA de Nantes, du 30 mars 2010 , $n^{\circ} 081045$ qui relève une telle erreur manifeste du maire n'ayant pas retiré un permis tacite anormalement accordé à proximité de la digue de La Faute-sur-Mer jugée dangereuse (et à juste titre, Ô combien!) par le préfet.

33. Le Conseil d'État a ainsi réitéré, en 2001 et 2002, son avis de 1997 , et appliqué aux PPR dans plusieurs arrêts, suivi par les Cours Administratives d'Appel et les Tribunaux administratifs, la qualification de document ou de dispositions d'urbanisme. Pour un exposé complet de l'évolution jurisprudentielle voir le commentaire, par Frédéric Dieu, Commissaire du Gouvernement (TA de Nice), de l'arrêt CAA Bordeaux du 31 août 2006 Sté arboricole et fruitière de l'Agenais nº 04BX 00807. de documents d'urbanisme des plans de servitudes, le principe de compatibilité entre les documents d'urbanismes d'un même territoire devant obliger les communes à la révision de leur PLU ${ }^{34}$.

Remarquons que le législateur n'a jamais entendu fractionner la notion de documents d'urbanisme et n'a pas remis en cause la définition donnée par le Conseil d'État depuis $1997^{35}$. Il s'est contenté de donner une liste des documents d'urbanisme (L. 121-1) qui répondent à la définition donnée par le juge car ce sont bien des « documents élaborés à l'initiative des collectivités publiques et ayant pour objet de déterminer les prévisions et règlements touchant à l'affectation et à l'occupation des sols opposables aux personnes publiques et privées » (Bonichot, 1997; Le Cornec, 1997 ; Lebreton, 1998, 2002). De notre point de vue, la liste est ouverte puisque l'article L. 121-1 ne cite toujours pas le SMVM, qui a valeur de DTA ${ }^{36}$. Dans le même sens, il est à noter que l'administration locale a déjà intégré cette qualification des PPR par le juge ${ }^{37}$.

Le nouveau dispositif de la loi du 12 juillet 2010 est assez subtil pour contourner cette difficulté créée par le principe de séparation des législations. Au final ce n'est pas le PPRN qui impose la compatibilité au PLU mais le plan de gestion des

RDI 2007 page 103. Voir notamment : CAA Bordeaux 4 novembre 2004 $\mathrm{n}^{\circ}$ 02BX00258 Préfet de la Charente-maritime; TA Amiens 23 avril 2007, nº 0601149 (Erreur manifeste d'appréciation d'un zonage du PLU). A contrario : CAA Nantes 26 décembre 2003, nº 02NT00213 SCI la Petite Prise à La Faute-sur-Mer.

34. «Considérant que la société requérante ne peut utilement se prévaloir à l'encontre du plan de prévention des risques naturels d'une éventuelle discordance entre les prescriptions attachées au zonage dudit plan et celles attachées au zonage du plan local d'urbanisme, dès lors que [...] le plan, annexé au PLU devient, de ce fait opposable à toute demande relative à l'occupation ou l'utilisation du sol fondée sur le code de l'urbanisme. [...] Considérant qu'il est de la nature même de ce document d'urbanisme qu'est le Plan de prévention des risques naturels... » CAA Bordeaux, 30 juin 2008 n $^{\circ}$ 5 BX01417 SA Bonne Anse Plage camping caravaning. On ne peut mieux dire l'obligation de cohérence des deux documents.

35. L'annexe du rapport sénatorial comportant les auditions par la mission nous disposons d'un appareil doctrinal important notamment par les avis donnés par M. Raymond Léost, responsable juridique de France nature environnement, M. Jean-Bernard Auby, professeur des universités à Sciences Po, M.-Y. Jégouzo, professeur agrégé de droit public à Paris.

36. CE, 7 juillet 1997, Mme Madaule, $n^{\circ} 170375$, et Association « Sauvegarde de l'étang des mouettes et de l'environnement ", n 70406 BJDU 5/97 p. 315. Plus le décret du 20 avril 1995 approuvant le schéma du bassin de Thau, JO, 21 avril. Les SMVM fixent : « Les orientations fondamentales de la protection, de l'exploitation et de l'aménagement du littoral. » Ils ont la valeur de prescriptions d'aménagement et d'urbanisme. Ils sont associés aux SCOT depuis la loi d'urbanisme de 2003.

37. Le site de la préfecture de l'Oise indique, dans sa présentation de la " galaxie de documents s'articulant autour des PLU et des Scot », le PPR comme un document d'urbanisme opposable si annexé au PLU et sur la base de l'avis du CE du 3 décembre 2001 (DDE Oise, Les feuillets de l'Oise, $n^{\circ} 40$, mai 2006). 
risques d'inondation pris en application de l'article L. 566-7 du code de l'environnement ${ }^{38}$. Ce plan de gestion élaboré au niveau du bassin versant et intégré dans le SCOT, a un contenu de même nature que le SMVM; il est élaboré selon une procédure conforme au modèle d'élaboration des documents d'urbanisme et de la même façon, il impose la mise à jour des PLU dans les trois ans. Il n'y a donc aucune difficulté à le qualifier de plan d'urbanisme supérieur et impératif pour les communes.

Si le PPRN est amélioré notamment dans sa procédure de révision et de modification par l'article 222 de la loi, on remarque qu'il n'est pas cité dans cet article 221 qui modifie le chapitre VI du code de l'environnement relatif à l'évaluation et gestion des risques d'inondation. Il faut comprendre que le PPRN reste un outil pour tous les risques naturels et aussi pour les risques en zone inondables tant que ces derniers n'auront pas été traités par le dispositif des nouveaux articles 221 et suivants de la loi. Mais ensuite c'est un régime spécifique de documents d'urbanisme, issus d'une élaboration conjointe avec les collectivités territoriales et les établissements de bassins, qui permettra de prévenir ce risque spécifique. Ce qui peut se justifier à la fois par l'exposition majeure de cinq millions de Français et par l'intégration de la directive européenne de 2007. Les eaux marines côtières étant soumises au régime de la loi sur l'eau et la gestion intégrée des zones côtières imposant d'inclure les submersions marines dans ce régime, les communes littorales doivent s'attendre à une révision systématique de leurs documents d'urbanisme.

38. Art. L. 123-1-10 : «Le plan local d’urbanisme doit également, s'il y a lieu, être compatible avec les objectifs de gestion des risques d'inondation définis par les plans de gestion des risques d'inondation pris en application de l'article L. 566-7 du code de l'environnement, ainsi qu'avec les orientations fondamentales et les dispositions de ces plans définies en application des 10 et 30 du même article L. 566-7, lorsque ces plans sont approuvés.

«Lorsqu'un plan de gestion des risques d'inondation est approuvé après l'approbation d'un plan local d'urbanisme, ce dernier doit, si nécessaire, être rendu compatible dans un délai de trois ans avec les éléments mentionnés au premier alinéa du présent article.

«Dans ce cas, et par dérogation aux dispositions de l'article L. 123-1-9 du présent code, le plan local d'urbanisme n'a pas à être compatible avec les orientations fondamentales relatives à la prévention des inondations définies par les schémas directeurs d'aménagement et de gestion des eaux en application de l'article L. 212-1 du code de l'environnement. »
La prévention par la police de la construction en zone de risque

Si le juge judiciaire doit déterminer la responsabilité pénale des autorités de police, c'est le juge administratif qui détermine les devoirs de ces autorités et leurs conséquences éventuelles sur la responsabilité de l'État et des communes (Calderaro, 2001; Godbillon, 1999). Le schéma est assez simple : le maire ne peut délivrer un permis de construire en méconnaissance des périmètres et des règles de servitude régulièrement applicables par un PPRN et lui, comme le préfet, peuvent invoquer l'article R. 111-2 du code de l'urbanisme soit pour refuser une demande y compris dans une zone constructible au PLU, soit pour l'assortir de prescriptions utiles à réduire les risques pour les nouveaux habitants. L'objectif de sécurité de la police municipale générale, comme celui de la police spéciale des constructions, devant aboutir à un résultat effectif de protection de la population. Ce mécanisme permet d'appliquer un PPRN qui serait plus récent qu'un PLU avec lequel il serait en contradiction. Il permet aussi, en l'absence de PPRN de se fonder sur la réalité du risque démontré par les études et l'expérience pour refuser une construction supplémentaire. Mais bien entendu il ne joue qu'en présence d'un projet nouveau exigeant une autorisation administrative. C'est pourquoi le juge administratif intervient par le biais de l'exception d'illégalité du document d'urbanisme. Une véritable politique jurisprudentielle s'est développée notamment dans les Cours administratives de Nantes et de Bordeaux pour invoquer l'erreur manifeste d'appréciation lorsque les autorités ne font pas application de R. 111-2.

À propos d'un certificat d'urbanisme négatif, la CAA de Bordeaux confirme l'autonomie complète de l'article R. 111-2 qui, dans toutes les circonstances, en présence ou en l'absence de document local d'urbanisme, est un impératif pour l'administration chargée de la sécurité. Ce qui sera repris par le Conseil d'État en 2007 sur un arrêt de 2004 de la même Cour concernant la commune des Portes en Ré ${ }^{39}$. La CAA de Nantes, en 2005, dira que même en l'absence de PPRN opposable à la date de révision du PLU de Bourgneuf-en-Ré, le risque évident de submersion du marais Poitevin doit jus-

39. Et repris par le jugement du Tribunal Administratif de Nantes, M. et $M^{\text {me }}$ Bethon, précité. 
tifier une zone inconstructible, alors même que des constructions existent déjà et parce que les intérêts écologiques, paysagers et patrimoniaux se joignent à cette présence du risque pour que la protection soit impérative. Enfin la CAA de Bordeaux, qui est décidément la source principale de la jurisprudence en la matière et n'a jamais varié de sa ligne, non seulement déduit en 2006, de la qualité de document d'urbanisme d'un PPR, qu'il est soumis aux règles de procédure des articles L. 600-1 à 4 du CU mais surtout que ce PPR est un document supérieur au PLU en édictant des servitudes de zones inondables supérieures aux zones naturelles constructibles pour les loisirs (dites NALi). La même Cour confirmant sa position en 2008 en rappelant que le document d'urbanisme PPRN est, de par l'article L. 562-1 du code de l'environnement dont il est issu, complètement opposable au PLU même si le zonage de celui-ci le contredit.

Une bonne application des règles dégagées par le juge aurait probablement suffi à empêcher l'accroissement récent de l'exposition au risque de submersion. Mais, outre les fragilités d'un système qui ne s'applique qu'en aval, à la décision individuelle, il faut se rappeler la forte résistibilité des politiques communales aux plans de prévention et l'indifférence souvent constatée des communes aux avertissements des préfets, ou encore l'aveuglement des services de l'État délégués par certaines communes à l'instruction de leurs permis de construire. L'injonction d'une plus grande sévérité et vigilance à l'application de R. 111-2, faite par le gouvernement aux préfets à travers la circulaire de 2007, arrivait bien tard et ne pouvait concerner les centaines de maisons déjà construites en zone inondable.

On voit bien alors que la démarche de la loi Grenelle est meilleure car elle pose la question du bon niveau territorial de la décision d'urbanisme face à la pression de la rente immobilière. Mais elle dispose pour l'avenir et le délai sera long avant que les plans de gestion des risques d'inondation soient opposables. Il faudrait donc à plus court terme se tourner vers des moyens opérationnels dont la Mission parlementaire trace les grandes lignes.

\section{La prévention par l'aménagement opérationnel}

Le rapport de la mission parlementaire met en relief la mauvaise gestion de l'événement à chacune de ses cinq étapes : une prévision qui n'a pas permis d'anticiper correctement les risques à terre, une vigilance insuffisamment opérationnelle, une prévention incomplète du risque de submersion marine, une occupation des sols exposant au risque d'inondation, un entretien très inégal des digues. Les outils opérationnels défaillants pour chacune de ces étapes sont un enjeu primordial de la réforme. Les deux plus importants sont sans doute le système de digues et la gestion foncière d'un espace trop convoité.

\section{La prévention par les travaux de défense contre la mer}

Rompues, submergées par des vagues plus hautes, les digues, très hétérogènes dans leur consistance et leur continuité ont été deux causes aggravantes du malheur, par leur inefficacité face aux vagues et par le barrage qu'elles ont opposé à l'évacuation des eaux. De plus, les étiers n'étant pas munis de portes à la mer, comme dans le marais de Dol par exemple, les petits cours d'eau côtiers ont servi de porte d'entrée à l'eau salée qui est restée derrière les digues, dont beaucoup ont été supprimées ces dernières années par les remembrements, au-delà d'une journée et assez longtemps pour stériliser les champs ${ }^{40}$.

La tempête de 1999 avait mis ce problème en évidence et les digues ou améliorations en projet n'ont pas été réalisées, preuve qu'il s'agit moins d'une absence de volonté que d'une organisation bloquée à l'origine de l'inaction.

À peine $20 \%$ du linéaire des digues pourrait résister à un événement centennal ce qui s'explique par le fait que la loi de 1807 et les lois créant les associations de propriétaires chargées de les construire avaient essentiellement des visées agricoles. Le système de propriété et de gestion de ces digues, qui est décrit comme totalement anarchique par le rapport de la mission, ne permet pas un bon entretien par les propriétaires, ne favorise pas une action concertée des structures intercommunales ni une

40. Selon le rapport, « on estime que 75 kilomètres de digues sur 103 kilomètres sont à reconstruire ou à consolider en Vendée et 120 kilomètres de digues sur 224 kilomètres en Charente-Maritime ». 
intervention forte des conseils généraux pour les transformer en véritables murs contre la montée des eaux au droit des zones urbaines. Car il faut distinguer entre ces zones urbaines, inconnues à l'époque de la construction des digues et les zones agricoles où l'on peut conserver des digues peu élevées en organisant le recul stratégique et la compensation d'éventuelles pertes de rendement des surfaces par les fonds de réparation des calamités agricoles.

En outre on apprend que l'État s'est désintéressé des digues et a laissé les départements en financer l'entretien. Manque d'argent, coordination défectueuse, conflits d'intérêts entre associations de propriétaires et collectivités locales, négligence du risque, mais surtout, incompréhension de la complexité foncière et absence de débat politique et de choix sur les intérêts à défendre par des digues plus sérieuses ou une autre gestion de l'espace naturel littoral, sont à l'origine de cette grande indifférence au risque littoral.

Les propositions de la mission parlementaire font ressortir l'importance des enjeux « financiers » du plan digue annoncé par le gouvernement.

En premier lieu, la mission semble fascinée par l'exemple néerlandais et approuve l'idée du Ministre d'engager une dépense de 470 millions d'euros sur six ans soit l'équivalent d'une année d'investissement aux Pays-Bas, dont le littoral s'étend sur 350 kilomètres alors que celui de la France compte 5853 kilomètres. En annonçant un «plan digues » national de presque 4 milliards, le ministre sort de l'épure Xynthia, alors que c'est le littoral atlantique qui est le plus menacé par le changement climatique, que les segments côtiers ne sont pas tous prioritaires et qu'il ne s'agit pas de corseter notre littoral d'un "mur de l'Atlantique » à la néerlandaise! Ces chiffres sont avancés sans étude précise des protections efficaces qui seraient placées dans des segments stratégiques du littoral (portes à la mer, protection de zones urbaines, blocage des linéaires exposés à la houle de sud-ouest, etc.), sans inventaire des besoins les plus cruciaux ni des solutions alternatives à l'élévation de digues artificielles. D'ailleurs l'estimation ramenée aux départements les plus touchés atteindrait les 200 millions. L'enjeu de la ressource est donc dramatisé par cette imprécision des échelles de chiffrage et l'on songe évidemment à « gonfler le fonds Barnier » dont on sait pourtant qu'il ne pourra tout satisfaire puisqu'il augmentera au maximum de 100 millions par an tout en devant assumer l'indemnisation des zones de solidarité! La mission en conclut raisonnablement que le programme devra s'étaler sur le long terme et ne pas se contenter de replâtrage, ce qui suppose une intervention sur les segments prioritaires du littoral pour prévenir d'autres catastrophes ${ }^{41}$.

Le second enjeu est donc celui de l'implication forte de l'État dans un tel programme, ce qui suppose une réforme profonde du système de la loi de 1807. Déjà au titre de l'article 211-7 du code de l'environnement les collectivités et leurs groupements peuvent se charger des ouvrages de lutte contre les inondations et les submersions. La loi Grenelle II organise aussi une intervention des établissements publics de bassin ${ }^{42}$ qui peut comporter la définition des travaux mais ne leur donne pas la maîtrise d'ouvrage du plan digue ${ }^{43}$. On reste donc très loin d'une réorganisation des pouvoirs en la matière.

Enfin, et c'est un enjeu politique puisqu'il s'agit de la participation des habitants à leur propre défense, la mission propose d'étudier l'affectation d'une ressource fiscale nouvelle (taxe additionnelle à la taxe foncière sur les propriétés bâties ou non bâties) ou la perception d'une redevance de participation aux dépenses engagées sur les ouvrages. Le principe en est sain car il fait penser aux participations prévues par le code de l'urbanisme exigibles pour les ouvrages publics nécessités par une construction nouvelle. Mais pourquoi s'en tenir à une redevance perçue au titre de l'article L. 211-7 et ne pas proposer d'intégrer le financement des digues comme participation spécifique d'urbanisme après l'article

41. Le rapport cite la secrétaire d'État à l'écologie : « Le plan digues sera une anticipation de la stratégie nationale des inondations sur certains secteurs prioritaires. La liste des territoires à risques importants d'inondation doit en effet être arrêtée début 2012. Certains secteurs prioritaires sont déjà connus (zones prioritaires des plans grands fleuves, zones disposant d'un programme d'action et de prévention des inondations (PAPI) opérationnel ou en cours de réalisation, zones "jaunes" de Xynthia). »

42. Pour l'établissement des plans de gestion des risques qui peuvent comprendre un programme de travaux : L 566-7 nouveau du code de l'environnement : « Le plan de gestion des risques d'inondation peut identifier les travaux et mesures relatifs à la gestion des risques d'inondation qui doivent être qualifiés de projet d'intérêt général en application de l'article L. 121-9 du code de l'urbanisme, et fixer les délais de mise en œuvre des procédures correspondantes par l'autorité administrative compétente.

43. Code de l'environnement Art. L. 566-10 : Les établissements publics territoriaux de bassin mentionnés à l'article L. 213-12 assurent à l'échelle du bassin ou sous-bassin hydrographique de leur compétence la cohérence des actions des collectivités territoriales et de leurs groupements visant à réduire les conséquences négatives des inondations sur les territoires mentionnés à l'article L. 566-5, par leur rôle de coordination, d'animation, d'information et de conseil pour des actions de réduction de la vulnérabilité aux inondations. 
L. 332-8 du code de l'urbanisme ${ }^{44}$ ? Est-ce parceque le bénéficiaire qui serait chargé des digues n'est toujours pas clairement déterminé?

Inventer de nouveaux outils opérationnels ou accélérer la mise en œuvre d'outils existants

Deux groupes de mesures de bon sens sont proposées par la mission parlementaire. Il s'agit d'utiliser tous les outils fonciers disponibles soit pour permettre aux habitants exposés de s'extraire des zones dangereuses, soit pour créer des zones naturelles jouant le rôle de tampon. Il faut ensuite améliorer les outils réglementaires en intégrant mieux le risque dans la loi littoral et en concevant les plans de gestion des risques comme des schémas d'aménagement des zones littorales à risques tels qu'ils sont proposés par la mission parlementaire, qui seraient en quelque sorte les documents guides de ces interventions opérationnelles. On est aussi étonné du silence des rapports sur l'impact des reculs stratégiques sur l'urbanisation de la zone littorale. Est-ce forcément la commune sinistrée qui doit organiser sur son seul territoire l'accueil des personnes déplacées? Ou bien la coopération intercommunale ne devrait-elle pas déjà se mettre en place pour organiser un aménagement urbain en profondeur dans l'esprit de la loi littoral? Ce qui supposerait sans doute une adhésion des communes de second rang à l'application de la loi littoral. Peut-on suggérer au Législateur de modifier cette loi pour l'adapter à ces territoires qui ne sont plus contigus de la mer et pour lesquels toutes les contraintes de la loi littoral ne seraient pas forcément imposables, ce qui faciliterait leur adhésion et impliquerait les cantons dans la gestion intégrée de la zone côtière?

Pour ce qui est des mesures foncières, faire intervenir les droits de préemption de la SAFER, des espaces naturels sensibles des départements, du Conservatoire du littoral et des communes par le droit de préemption urbain est une solution nécessaire. Mais on peut sans doute aller plus loin en développant les procédures d'acquisitions amiables et en introduisant un droit de délaissement dans les zones dangereuses. De cette façon la solidarité nationale jouerait préventivement. À cela que pro-

44. La mission propose de moduler le taux de la TLE (taxe locale d'équipement), ce qui constitue une des formes de participation mais sujette à exonérations et reports alors qu'ici l'égalité devant l'exposition au risque devrait en faire une participation forfaitaire. pose la mission, on devait ajouter une réflexion sur le statut de la propriété privée face au risque et sur l'affectation des propriétés publiques, notamment par la création des zones naturelles assumant à la fois protection et mise en valeur écologique. Si le Conservatoire du littoral prévoit toujours un plan de gestion de ses propriétés, ce n'est pas si évident pour les terrains achetés par les départements ou les communes. Il faudrait donc un plan global d'aménagement et de gestion des espaces naturels distribuant les rôles entre les différents acquéreurs publics et déterminant peut-être un gestionnaire unique dans chaque zone homogène. Nous disposons pour cela d'un excellent moyen, la zone d'aménagement concertée que les articles L. 300-1 et suivants du code de l'urbanisme autorisent pour la sauvegarde et la mise en valeur des espaces naturels. La coordination de ces opérations pose cependant le problème du territoire d'intervention et de sa gouvernance.

\section{Coordonner les interventions publiques}

sur des territoires pertinents

L'intervention opérationnelle sur le triple plan des ouvrages publics, de l'action foncière et de la gestion des milieux, ne peut se fonder sur le découpage administratif des territoires qui ne correspondent ni au terrain des risques ni aux moyens nécessaires. C'est le risque qui devrait imposer sa délimitation du territoire.

La mission parlementaire fait grand cas de l'expertise des élus locaux et de la nécessité de leur confier l'organisation des schémas locaux en concertation avec l'État et non l'inverse. Mais sont-ils à même de s'organiser alors que les syndicats intercommunaux ont le plus grand mal à coopérer entre eux de part et d'autre d'une même rivière? N'est-ce pas la loi qui devrait imposer la définition des territoires pertinents? On en prend le chemin avec la procédure assez complexe d'élaboration des plans de gestion des risques alors que le rôle de l'établissement public de bassin n'est pas très bien défini. Ce qui nous donne encore un sentiment d'inachevé dans la démarche du législateur. Donc, une fois de plus tout tiendra à la capacité des préfets de convaincre ou d'imposer la réflexion et la décision à ce bon niveau de coopération et à la capacité de l'État de soutenir ses préfets dans cette tâche. 
Un autre aspect des propositions d'organisation de la gouvernance paraît inquiétant, celui de la prise en compte des citoyens. Si leur participation est prévue dans l'élaboration des plans de gestion des risques et selon le modèle désormais bien établi par l'application de la directive Plans programmes, le niveau de prise ne compte de leur avis semble éloigné du terrain alors que les habitants exposés aux risques on tendance à ne considérer que leur commune. Va-t-on inciter à la création d'associations citoyennes à l'échelle des bassins versants?

L'enjeu démocratique est considérable car sans acceptation des projets par le public rien ne pourra se faire et on continuera de voir des installations dans les zones à risque. Pourvu que l'on n'ait pas, dans un proche avenir, à parodier encore Sganarelle : «Mais qu'allaient-ils donc faire dans cette galère? »

\section{Lexique des principaux sigles}

DTA : Directive territoriale d'aménagement.

PLU : Plan local d'urbanisme

SCOT : Schéma de cohérence territoriale

SMVM : Schémas de mise en valeur de la mer PPR, PPRN : Plan de prévention des risques (naturels) PPRI : Plans de prévention des risques d'inondation

TA : Tribunal administratif

CE : Conseil d'État

CAA : Cour administrative d'appel

\section{Bibliographie}

Agostini F.-R., 2000. Les droits de la partie civile dans le procès pénal, Rapport annuel de la Cour de Cassation, [http:// www.courdecassation.fr/publications Rapport 2000].

Anziani R., 2010. Les conséquences de la tempête Xynthia, Rapport du Sénat $n^{\circ}$ 647, Paris, vol. 1 \& 2 (7 juillet 2010), [http://www.senat.fr].

Auby J.-B., 2005. Le droit administratif dans la société du risque: quelques réflexions, Etudes et documents du CE, Rapport annuel du CE, Section du rapport et des études, Paris, La Documentation Française, 351 p.

Bentoglio G., Betbèze J.-P. (dir.), 2004. L'État et l'assurance des risques nouveaux - Exercice prospectif, Commissariat général au Plan 2004, Paris, La Documentation Française, 2005, $121 \mathrm{p}$.

Bonichot J.-C., 1997. Conclusion sous Avis du CE Sect. 17 janvier 1997, Association de défense du site de l'environnement de Gallui, LPA 1997, n² 28, p. 17.

Calderaro N., 2001. Le juge administratif et les risques naturels, Revue française de droit administratif (RFDA), 895 p.

Cimelière O., 2010.Victime je pense, donc victime je suis, Le Blog du Communiquant, (8 nov. 2010). [http://www. leblogducommunicant2-0.com].

Conseil d'État, 2005. Rapport d'activité pour 2004, Études et Documents du Conseil d'État, Paris, La Documentation Française, (n. p.).

Danet J., 2006. Justice pénale, le tournant, Paris, Gallimard, coll. « Folio actuel », 393 p.

DANET J. (dir.), 2004. Le nouveau procès pénal après la Loi Perben II, Paris, Dalloz, coll. « Dossiers Journées d'Études Dalloz », 591 p.

Dieu F., 2007. L'application des dispositions de l'article L 600-1 aux PPRN ou du caractère attractif des documents d'urbanisme, Revue de droit immobilier, p. 103.

Favro K., 2009. Risques et territoires, plaidoyer pour une méthode globale d'analyse des risques, Préventique-Sécurité, (juillet-août 2009), n 106, p. 30-36.

Godbillon B., 1999. L'autorisation de construire et le risque naturel : Le devoir de vigilance en droit de l'urbanisme, Actualité Juridique de la Propriété Immobilière, (10 septembre 1999), p. 68.

Huglo C., 2010. Catastrophes écologiques : le procès pénal doit-il être la règle?, Les Échos, n 20608, (4 février 2010), p. 11.

Jeanneaux P., Sabau C., 2009. Conflits environnementaux et décisions juridictionnelles : que nous apprend l'analyse du contentieux judiciaire dans un département français (Puy de Dôme) ?, Vertigo, vol. 9, n 1, [http://vertigo.revues.org/8412].

Lebreton J.-P., 1998. Documents d'urbanisme : émergence d'une notion jurisprudentielle, Revue de l'Association française de droit de l'urbanisme et de l'habitat (AFDUH), n 12 , p. 33.

Lebreton J.-P., 2002. Note sous Conseil d'État, avis, 12 juin 2002, Préfet de la Charente-Maritime, requête n 244.634, AJDA (Actualité juridique. Droit administratif), $\mathrm{n}^{\circ}$ 16, p. 1081 .

Le Cornec E., 1997. Les critères du document d'urbanisme: une analyse critique : Revue française de droit administratif (DA), n 17, p. 4.

Pontier J.-M., 2003. La puissance publique et la prévention des risques, AJDA (Actualité juridique. Droit administratif), $\mathrm{n}^{\circ} 33$, p. 1752-1761.

SÉCHet G., 2004. Quel temps! Chronique de la météo de 1900 à nos jours, Paris, Henné, $256 \mathrm{p}$. 